\title{
Structure and Chemistry of Oxide Surface Reconstructions in III-Nitrides Observed using STEM EELS
}

J. Houston Dycus ${ }^{1}$, Kelsey J. Mirrielees ${ }^{1}$, Everett D. Grimley ${ }^{1}$, Rohan Dhall ${ }^{1}$, Ronny Kirste ${ }^{2}$, Seiji Mita ${ }^{2}$, Zlatko Sitar ${ }^{1}$, Ramon Collazo ${ }^{1}$, Douglas L. Irving ${ }^{1}$ and James M. LeBeau ${ }^{1}$

1. Department of Materials Science and Engineering, North Carolina State University, Raleigh NC, 27695-7907

2. Adroit Materials, Inc., 2054 Kildaire Farm Rd., Suite 205, Cary, North Carolina 27518, USA

While characterization in the bulk of materials has become thoroughly performed for many systems, surfaces often have reconstructions with dissimilar structure. Because of the deviation from parent structure, surfaces have been shown to exhibit different electronic states than bulk [1,2]. One important system that surfaces play an important role is in the family of III-nitrides with applications ranging from light emitting diodes to deep ultraviolet lasers for water purification; however, a strong understanding of surface features remains unresolved. Towards this end, many studies have been performed to provide information on reconstructions in III-nitride surfaces. Previously, XPS has found that oxygen is present near the c-plane surface. Knowing that oxygen is contained at the surface, many efforts using first principles density functional theory (DFT) have been performed with aims to uncover the origins of electronic properties such as the formation of the two-dimensional electron gas $[3,4]$. Although several reports have provided models for the structural configuration at the GaN/AIN surface, no direct evidence has clearly revealed the surface reconstruction for c-plane surfaces. For direct atomic structure identification, scanning transmission electron microscopy (STEM) should serve as an ideal tool.

In this presentation, we investigate the oxide surfaces of AlN and GaN crystals using STEM. With a combination of annular dark-field imaging (ADF) and electron energy-loss spectroscopy (EELS) the atomic bonding and chemistry of surface reconstructions are identified. First, we will discuss the important role of unique sample preparation conditions required for obtaining samples high quality samples. Once high quality samples have been prepared, we show that surfaces can be directly imaged. Figure 1 shows experimental and simulated annular dark-field images acquired along the [1120] and [1100] zone axes. A surface reconstruction occurs with an inversion from III-polar to N-polar at the final layer and a triplet serving as the inversion plane. We will then show EELS spectra across the surface that reveals the presence of oxygen in the final few layers, as shown in Figure 2 A-B. Further, by employing revolving STEM (RevSTEM), we will measure the distance for each unique site, Figure 2 C$\mathrm{D}$, to determine differences in local bonding environment in comparison to the bulk [5,6]. Using the experimentally determined structure as an input model for DFT simulations, we make energetic comparisons between the observed reconstruction and previously reported cases. Further, we look at the impacts of the reconstruction on properties such as band bending. Finally, we will discuss the implications of the results from this study on growth behavior and electronic properties for optoelectronic III-nitride materials. 
References:

[1] G. Z. Zhu, G. Radtke and G. A. Botton, Nature 490 (2012), p. 384-387.

[2] W. Xu et. al., Applied Physics Letters 109(20) (2016), p. 201601.

[3] Y. Dong, R. M. Feenstra and J. E. Northrup, Journal of Vacuum Science and Technology 24(4) (2006), p. 2080-2086.

[4] M. S. Miao, J. R. Weber and C. G. Van de Walle, Journal of Applied Physics 107 (2010), p. 123713

[5] J. H. Dycus et al, Microscopy and Microanalysis, 21 (2015), p. 946-952.

[6] X. Sang and J. M. LeBeau, Ultramicroscopy, 138 (2014), p. 28-35.

[7] JHD, EDG and JML acknowledge the Analytical Instrumentation Facility (AIF) at North Carolina State University. JHD and EDG acknowledge support by the National Science Foundation Graduate Research Fellowship (Grant DGE-1252376). Research was supported by the Air Force Office of Scientific Research (Grant No. FA9550-14-1-0182).

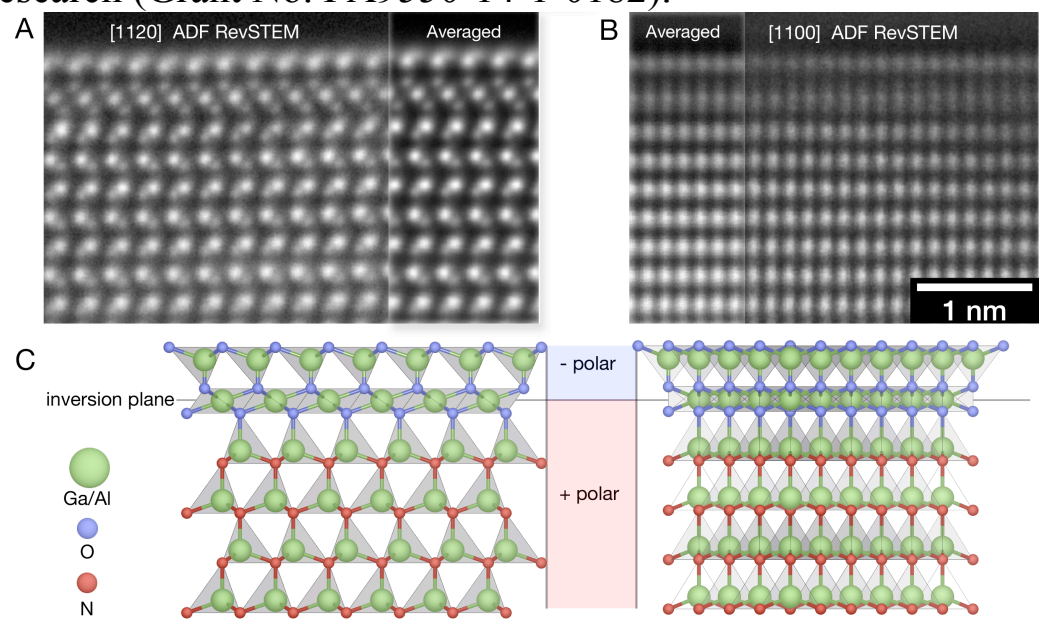

Figure 1. ADF imaging of an AIN c-plane surface using RevSTEM, template averaging and multislice simulations for [1120] and [1100] directions. The bottom shows the experimentally determined model.
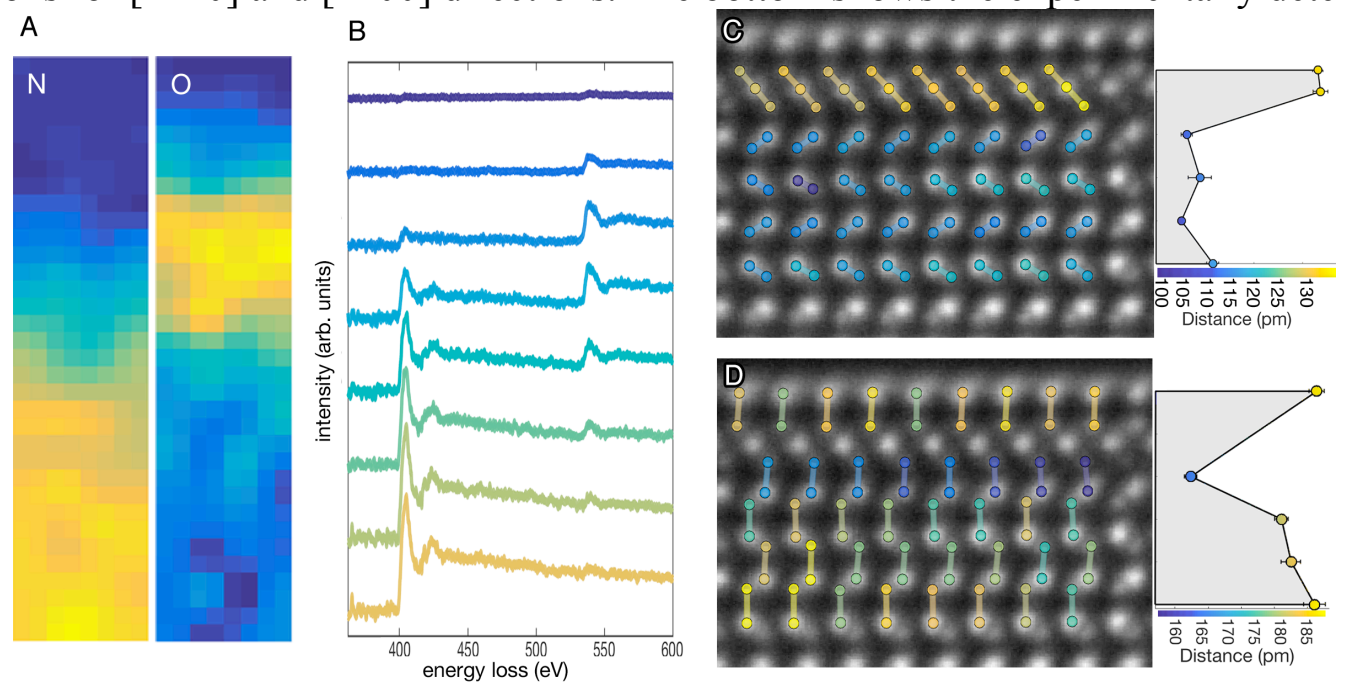

Figure 2. EELS (A-B) and distance (C-D) mapping for the AlN c-plane surface. Displayed EELS spectra are the integrated spectra for the corresponding rows in the adjacent EELS maps. Colors for measured intensities in (C-D) correspond to the distance from the color scale below the distance plots. Each point on the plot represents the average from each row of measured distances in the images. 